\section{OP0179 \\ DO DISTINCT PAIN PHENOTYPES HAVE DIFFERENT RISK OF KNEE REPLACEMENT: A 13.7-YEAR FOLLOW-UP STUDY?}

Feng Pan ${ }^{1}$, Jing Tian ${ }^{1}$, Ishanka Munugoda ${ }^{1}$, Stephen Graves ${ }^{2}$, Michelle Lorimer ${ }^{3}$, Flavia Cicuttini ${ }^{4}$, Graeme Jones ${ }^{1} .{ }^{1}$ University of Tasmania, Menzies Institute for Medical Research, Hobart, Australia; ${ }^{2}$ Australian Orthopaedic Association National Joint Replacement Registry (AOANJRR), Adelaide, Australia; ${ }^{3}$ South Australian Health and Medical Research Institute (SAHMRI), Adelaide, Australia; ${ }^{4}$ Monash University Medical School, Department of Epidemiology and Preventive Medicine, Melbourne, Australia

Background: Pain is the main impetus for osteoarthritis $(O A)$ patients to seek healthcare including joint replacement. The pain experience is very heterogeneous and affected by factors across multiple domains-peripheral, psychological, and neurological, which suggests the existence of homogenous subgroups/phenotypes within OA patients with pain. We recently identified three pain phenotypes using a wide spectrum of factors including main pain dimensions (structural damage on MRI, body mass index (BMI), comorbidities, psychological and multi-site pain (a surrogate of neurological factor)).

Objectives: To examine whether the risk of knee replacement (KR) varied when these three pain subgroups were compared.

Methods: 1099 participants (mean age 63 years; range 51-81 years) from a population-based cohort study were recruited at baseline. 875,768 and 563 participants attended years 2.6, 5.1 and 10.7 follow-up, respectively. Demographic, psychological, lifestyle and comorbidities data were obtained at baseline. T1-weighted or T2-weighted MRI of the right knee was performed to measure knee structural pathology-cartilage defects, bone marrow lesions (BMLs) and effusion-synovitis at baseline. Knee pain was assessed using the Western Ontario and McMaster Universities Osteoarthritis Index (WOMAC) pain questionnaire at each time-point. Presence of pain (yes/no) in the neck, back, hands, shoulders, hips, knees and feet was also assessed by questionnaire at each time-point. KR up to 13.7 years after recruitment was identified by linking to the Australian Orthopaedic Association National Joint Replacement Registry. Latent class analysis was used to differentiate pain phenotypes by considering sex, BMI, emotional problems, education level, comorbidities, number of painful sites and knee structural damage on MRI. Log-binomial regression was used to evaluate the association between three pain phenotypes and risk of KR.

Results: 963 participants were included in the analysis (BMI $27.7 \mathrm{~kg} / \mathrm{m}^{2}, 50 \%$ female). Three distinct phenotypes were identified: Class 1: high prevalence of emotional problems and low revalence of structural damage (25\%); Class 2 : high prevalence of structural damage and low prevalence of emotional problems (20\%); Class 3: low prevalence of emotional problems and low prevalence of structural damage (55\%). Participants in Class 1 had greater pain severity than those in Class 2 and 3. During a follow-up of 13.7 years, There were 46 right and 51 left TKR's in 79 participants In multivariable analyses, participants in Class 1 and 2 had higher risk of requiring $K R$ on the right [Class 1 vs. Class 3 : relative risk (RR) 6.66, 95\%confidence interval (Cl) 2.17-20.49; Class 2 vs. Class 3: RR 14.14, 95Cl\% 4.85-41.22], left (Class 1 vs. Class 3: RR 2.75, 95\%Cl 1.22-6.19; Class 2 vs. Class 3: RR 5.46, 95Cl\% 2.64-11.32), and any knee joints (Class 1 vs. Class 3: RR 3.65, $95 \% \mathrm{Cl} 1.88-7.09$; Class 2 vs. Class 3: RR $6.81,95 \mathrm{Cl} \%$ $3.68,12.60$ ) compared to Class 3 , but the associations were stronger with Class 2 than Class 1.

Conclusion: Participants with distinct pain phenotype groups have different risks of KR. This suggests that the identified phenotypes reflect distinct clinical subgroups with different prognoses. As expected the highest risk of $\mathrm{KR}$ was found in those with the most structural damage (Class 2). However, those participants with low structural damage have an increased risk of KR when they have high compared to low emotional problems, indicating that selection of subjects for KR could be improved by screening out those in Class 1 .

Disclosure of Interests: None declared

DOI: 10.1136/annrheumdis-2019-eular.6588

\section{OP0180 \\ LOW-DOSE PREDNISOLONE IN PATIENTS WITH HAND OSTEOARTHRITIS (HOPE): RESULTS FROM A RANDOMISED DOUBLE-BLIND PLACEBO- CONTROLLED TRIAL}

Féline Kroon ${ }^{1}$, Marion Kortekaas ${ }^{1}$, Annelies Boonen ${ }^{2,3}$, Stefan Böhringer ${ }^{4}$, Monique Reijnierse ${ }^{5}$, Frits Rosendaal ${ }^{6}$, Naghmeh Riyazi ${ }^{7}$, Mirian Starmans ${ }^{8}$, Franktien Turkstra ${ }^{9}$, Jende van Zeben ${ }^{10}$, Cornelia Allaart ${ }^{1}$,

Margreet Kloppenburg ${ }^{1,6} .{ }^{1}$ LUMC, Rheumatology, Leiden, Netherlands;

${ }^{2}$ Maastricht University Medical Center, Rheumatology, Maastricht, Netherlands;

${ }^{3}$ Care and Public Health Research Institute, Maastricht, Netherlands; ${ }^{4}$ LUMC, Medical Statistics, Leiden, Netherlands; ${ }^{5}$ LUMC, Radiology, Leiden, Netherlands; ${ }^{6}$ LUMC, Clinical Epidemiology, Leiden, Netherlands: ${ }^{7}$ Haga Hospital, Rheumatology, The Hague, Netherlands; ${ }^{8}$ Zuyderland Medical Center Rheumatology, Heerlen, Netherlands; ${ }^{9}$ Amsterdam rheumatology and immunology center / Reade, Amsterdam, Netherlands; ${ }^{10}$ Sint Franciscus Vlietland Group, Rheumatology, Rotterdam, Netherlands

Background: Hand osteoarthritis $(\mathrm{OA})$ is a prevalent joint disease with high disease-burden in need for effective therapeutic options. Studies have shown that synovial inflammation is often present in hand $\mathrm{OA}$ and a main determinant of pain and radiographic disease progression.

Objectives: To investigate the efficacy and safety of short-term low-dose prednisolone in patients with painful hand OA.

Methods: This randomised, double-blind, placebo-controlled trial enrolled patients with painful hand OA, fulfilling American College of Rheumatology criteria, and signs of synovial inflammation. Patients with $\geq 4$ interphalangeal joints (IPJ) with osteoarthritic nodes, $\geq 1$ IPJ with soft swelling or erythema and $\geq 1$ IPJ with positive power Doppler signal (PDS) or synovitis grade $\geq 2$ on ultrasound, were eligible. Key exclusion criteria were chronic inflammatory rheumatic diseases, psoriasis, using immune modulating drugs within 90 days before baseline, and predominant thumb base pain. Eligible patients with visual analogue scale (VAS) finger pain $\geq 30 \mathrm{~mm}$, flaring $\geq 20 \mathrm{~mm}$ upon non-steroidal anti-inflammatory drug washout, were randomised to receive prednisolone $10 \mathrm{mg}$ daily for 6 weeks or placebo, followed by a two-week tapering scheme and 6 weeks without study medication. Outcomes were assessed at 2, 4, 6, 8 and 14 weeks. Primary endpoint was VAS finger pain at week 6 in intention-to-treat analysis. Secondary clinical endpoints included fulfilment of OMERACT-OARSI responder criteria, Australian/Canadian Hand OA Index (AUSCAN) pain/function, Functional Index for Hand OA (FIHOA), VAS patient global assessment, Short-Form 36 and grip strength. Imaging endpoints included ultrasound synovitis and PDS.
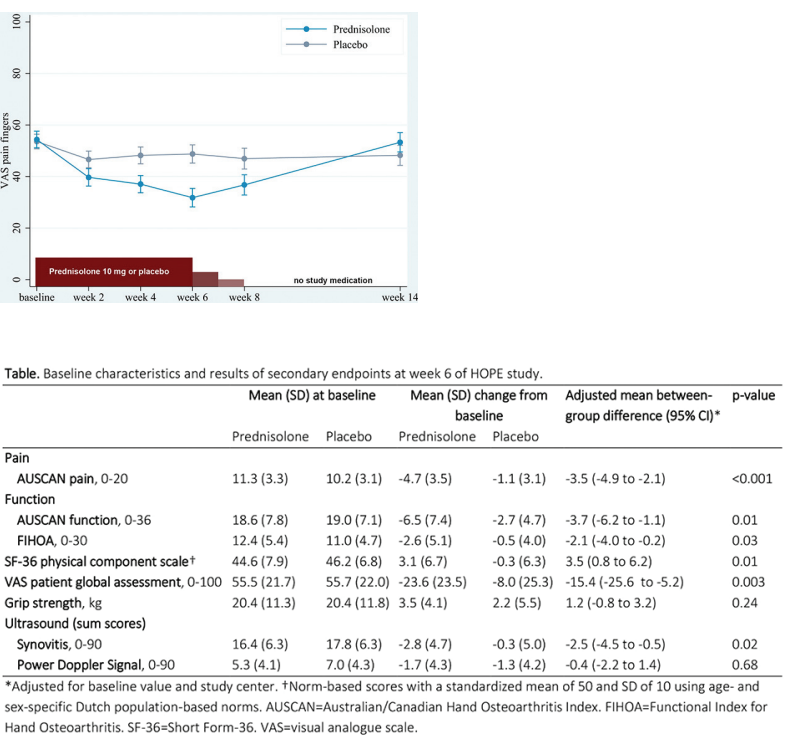

Results: Of 92 patients (mean (SD) age $63.9(8.8), 79 \%$ women) randomised to prednisolone $(n=46)$ or placebo $(n=46), 42$ patients in each group completed the study. Baseline characteristics were well-balanced between the groups. The mean (SD) change from baseline to week 6 in VAS finger pain was -21.5 (21.7) in the prednisolone and -5.2 (24.3) in the placebo group, with a mean between-group difference of -16.5 (95\% confidence interval (Cl) -26.1 to -6.9 ; figure). At week 6 , $33(72 \%)$ patients in the prednisolone versus $15(33 \%)$ in the placebo group fulfilled OARSI responder criteria (odds ratio $5.3,95 \% \mathrm{Cl} 2.0$ to $13.6, p=0.001$ ). In analogy with the primary endpoint, prednisolone was superior to placebo in most other secondary clinical endpoints (table). Ultrasound synovitis significantly improved at week 6 in the prednisolone compared to the placebo group, while no difference was observed in PDS (table). After tapering, between-group 